\title{
The Utility of Single Nucleotide DNA Variations as Predictors of Postoperative Pain
}

Gregory M. Jacobson ${ }^{1 *}$, Corinne J. Law ${ }^{2}$, Harriet Johnston ${ }^{1}$, Mark Chaddock ${ }^{3}$, Michal Kluger ${ }^{3}$, Raymond T.M. Cursons ${ }^{1}$ and Jamie W. Sleigh ${ }^{4}$

${ }^{1}$ Department of Biological Sciences, University of Waikato, Hamilton, New Zealand

${ }^{2}$ Department of Anaesthesia, Anglesea Hospital, Hamilton, New Zealand

${ }^{3}$ Waitemata DHB, Auckland, New Zealand

${ }^{4}$ Waikato DHB, Hamilton, New Zealand

\begin{abstract}
Objectives: Genetic variation is an important contributor to postsurgical pain and thereby analgesia requirements. A description of the potential predictive power of genetic variants in pain should instruct improvements in pain management postoperatively. We set out to examine whether a set of genetic variants in pain related genes would show any association with actual pain outcomes in a typical surgical population.

Methods: A candidate gene study was carried out in 135 surgical patients with 12 DNA variants (single nucleotide polymorphisms or 'SNPs') in known or putative pain pathway genes to detect associations with postoperative pain measured by a verbal rating score (VRS) and patient-controlled analgesia (PCA) usage rate. Standard PCR based molecular biology approaches were used.

Results: At 20-24h after surgery, patients with the 1032G/1032G variant pair for the A1032G variant of the potassium channel KCNJ6 gene had a slightly higher median VRS than those with 1032A/1032A or 1032A/1032G pairs $(p=0.04$; dominant genetic model). This small difference was most apparent in the orthopaedic surgery patients where the 1032G/1032G pair associated with VRS (median(interquartile range)) of 5(4-6) vs. 3(0.5-4) in $1032 \mathrm{~A} / 1032 \mathrm{~A}$ or $1032 \mathrm{~A} / 1032 \mathrm{G}$ groups. For PCA, patients with $3435 \mathrm{C} / 3435 \mathrm{C}$ or $3435 \mathrm{C} / 3435 \mathrm{~T}$ pairs for ATPdependent efflux pump gene $A B C B 1$ variant $C 3435$ T used PCA at a considerably higher rate of $0.89(0.07-1.66)$ $\mathrm{mg} \cdot \mathrm{h}^{-1}$ compared with just $0.11(0-0.52) \mathrm{mg} \cdot \mathrm{h}^{-1}$ for the $3435 \mathrm{~T} / 3435 \mathrm{~T}$ pair $(\mathrm{p}=0.03$; dominant model). A significantly higher usage rate was also detected for opioid receptor OPRM1 variant IVS2-691 with usage of 0.77(0.01-1.56) $\mathrm{mg} \cdot \mathrm{h}^{-1}$ for the IVS2C/IVS2C or IVS2C/IVS2G group vs. $0.24(0-1.26) \mathrm{mg}^{-\mathrm{h}^{-1}}$ in the IVS2G/IVS2G group $(p=0.04$; recessive model).
\end{abstract}

Conclusion: While this study has identified some significant statistical associations the potential utility of the studied DNA variants in prediction of postoperative pain and patient-controlled opioid analgesia requirements appears to be quite limited at present.

Keywords: OPRM1; ABCB1; Postoperative; Pain; Morphine; Analgesia

\section{Background}

Pain is a complex trait influenced by psychological, social, physical and genetic factors (reviewed [1]). Of the various factors that influence postsurgical pain, genetic variation plays an important role, with many gene variants contributing to the pain phenotype. Their cumulative small effects occur through alteration of protein expression level or amino acid sequence and thereby protein functions. Accordingly, DNA variants, in particular single nucleotide polymorphisms (SNPs), may produce differences in perception of acute pain [2] or the efficacy of postoperative analgesia [3].

A number of studies have identified genetic associations with acute pain. A survey of the literature for these associations highlights: 1) the ATP-binding cassette $(\mathrm{ABC})$ superfamily, subfamily B p-glycoprotein $170(A B C B 1)$ gene encoding an ATP-dependent drug efflux pump involved in multiple drug resistance. $A B C B 1$ is a major determinant of morphine bioavailability[4] and the 3435C allele of variant C3435T (rs1045642) in the gene associates with higher expression level and reduced morphine availability in the CNS. 2) The catechol-O-methyl transferase (COMT) gene encoding an enzyme that degrades several analgesic neurotransmitters, including noradrenaline and dopamine [7]. A well-studied variant in the gene, Val158Met (rs4680), encodes a valine-to-methionine variation that associates with reduced metabolic activity of the enzyme, as first reported by Scanlon and colleagues [8]. Reyes-Gibby [9] reports that cancer patients with the 158Val/158Val genotype required 63\% more morphine for efficient pain relief compared to patients with the $158 \mathrm{Met} / 158 \mathrm{Met}$ genotype. Another group has found that the 158Met variant was actually associated higher pain scores during painful postsurgical procedures (with morphine analgesia) [10]. 5) The $\mu$-opioid (OPRM1) gene encodes the $\mu$-opioid receptor and is a very well-studied candidate gene for pain association $[18,19]$. Gene expression from the $118 \mathrm{G}$ allele of OPRM1 variant A118G (rs1799971) - which codes for an asparagine to aspartic acid residue change- produces a receptor with reduced agonist-induced receptor signalling efficacy [20]. Various studies have shown association of this variant with increased pain scores and/or opioid analgesia requirements after surgery [21-24]. 6) The potassium inwardly-rectifying channel subfamily J, member 6 (KCNJ6) gene encoding an ATP-activated channel (GIRK2) that closes with increased intracellular ATP causing membrane depolarization and voltage-sensitive activation. GIRK2 is activated by several G-protein coupled receptors including opioid

${ }^{*}$ Corresponding author: Gregory M. Jacobson, Department of Biological Sciences, University of Waikato, Private Bag 3140, Hamilton, New Zealand, Tel: +64-21825333; Fax: +64-78384324; E-mail: Jacobson@waikato.ac.nz

Received March 05, 2014; Accepted April 11, 2014; Published April 14, 2014

Citation: Jacobson GM, Law CJ, Johnston H, Chaddock M, Kluger M, et al. (2014) The Utility of Single Nucleotide DNA Variations as Predictors of Postoperative Pain. J Anesth Clin Res 5: 401. doi:10.4172/2155-6148.1000401

Copyright: (C) 2014 Jacobson GM, et al. This is an open-access article distributed under the terms of the Creative Commons Attribution License, which permits unrestricted use, distribution, and reproduction in any medium, provided the original author and source are credited. 
receptors [26]. Nishizawa and co-workers [27] report that two SNPs in the gene - A1032G (rs2070995) and G-1250A (rs6517442) associate with postoperative rescue analgesia requirements. 7) The sodium channel protein type 9 subunit alpha ( $S C N 9 a)$ gene encodes a voltage-gated channel involved in nociception [29]. For $S C N 9 a$ variant Arg1150Trp (rs6746030), the channel protein expressed from the gene containing the $1150 \mathrm{Trp}$ variant shows enhanced levels of depolarizing activation [30].

Other studies have usually looked for associations of single DNA variants with chronic pain conditions, or for pain thresholds in laboratory tests of noxious stimuli on human volunteers. In this study we selected a panel of genetic variants, some with established and some with no previously suggested pain association, and measured whether this broader selection of DNA variants might contribute significantly to the variation in postoperative pain seen in a broad-based representative clinical sample of day-to-day adult postsurgical patients. We aimed to determine whether any of the DNA variants had real clinical usefulness for predicting high postoperative pain.

\section{Methods}

\section{Clinical}

We recruited participants who were undergoing elective nonemergent surgery (expected duration $<180 \mathrm{~min}$ ) and were aged 18$65 \mathrm{yr}$, were competent to consent, were able to comprehend the VRS and were able to activate the PCA device. Subjects with weight $>120$ $\mathrm{kg}$ and/or BMI $>35$, a preoperative diagnosis of malignancy, epilepsy, a neuromuscular disease, a previously documented psychiatric disease that required medication, pregnancy, a history of chronic opioid use or dependency or severe asthma (i.e. previously necessitating hospital admission), were excluded. Regional anaesthesia, neuraxial blockade and total intravenous anaesthesia was not permitted. Patients received standardised general anaesthesia consisting of an intravenous induction using propofol (1-3 mg.kg-1), muscle relaxant, and maintenance with 0.7-1.3MAC desflurane. The intraoperative opioid dosing regimen was 2 mcg. $\mathrm{kg}^{-1}$ fentanyl on induction, and a $2 \mathrm{mcg} \cdot \mathrm{kg}^{-1} \cdot \mathrm{h}^{-1}$ infusion from the time of surgical preparation to closure. All patients received $1 \mathrm{~g}$ paracetamol, $40 \mathrm{mg}$ parecoxib, $4 \mathrm{mg}$ dexamethasone, $0.5 \mathrm{mg}$ droperidol intravenously intraoperatively. Local anaesthetic was infiltrated to the wound at skin closure. Intravenous morphine PCA was provided postoperatively to achieve satisfactory patient comfort (1.5 mg doses, 5 min lockout interval, and $8 \mathrm{mg} \cdot \mathrm{hr}^{-1}$ maximum dose). Protocols received approval of the New Zealand Northern X regional ethics committee (\#NTX/09/06/047) with patients giving written informed consent.

Postoperative pain was quantified using a standard verbal rating scale (VRS) ranging from $0=$ "no pain at all" to $10=$ "the worst pain imaginable". The VRS was recorded upon awakening in the post anaesthetic care unit (post anaesthetic care unit - PACU; taken at rest) $\left(\right.$ VRS $_{\text {awake }}$ ) as well as for morning after surgery (at 20-24hrs) with activity $\left(\mathrm{VRS}_{\mathrm{d1A}}\right)$. For abdominal surgeries the pain score was for pain experienced during coughing and for orthopaedic surgeries the score was for pain during limb movement. Patient PCA usage rate over 20$24 \mathrm{~h}$ post-surgery was also recorded ( $\left.\mathrm{PCA}_{\mathrm{mg} . \mathrm{h}}{ }^{-1}\right)$.

\section{Selection of DNA variants (SNPs)}

In addition to the variants listed in the introduction (see points 1-7), and after examination of the literature, we chose to also examine: 1) an additional $A B C B 1$ gene variant, $T 1236 \mathrm{C}$, which has been associated with differential transport of various drug substrates of the ABCB1 efflux pump [6]; 2) an additional variant in OPRM1, IVS2-691, which may encode an altered receptor protein $[3,25]$. 2) a variant in the potassium voltage-gated channel, delayed-rectifier, subfamily $\mathrm{S}$, member 1 (KCNS1) gene, Ile40Val, associated with higher pain after noxious stimuli in healthy volunteers [28]); and finally: 3) variants of the cytochrome P450 oxidase CYP3A4 or CYP3A5 genes, namely C20230T in the CYP3A4 gene, and A6968G in the CYP3A5 gene.

\section{DNA extraction}

A $1 \mathrm{ml}$ venous blood sample was collected at the time of surgical closure and stored in $4 \mathrm{ml}$ of a DNA preservation solution (5M GITC, $20 \mathrm{mM}$ sarkosyl, $30 \mathrm{mM}$ trisodium citrate, $0.7 \%$ mercaptoethanol, $\mathrm{pH}$ 7). To extract the DNA, a $1 \mathrm{ml}$ sample of this preparation was collected into a new tube and a 1/10th volume of $3 \mathrm{M}$ sodium citrate and $0.5 \mathrm{ml}$ of phenol-chloroform ( $\mathrm{pH} 7$ ) were added and the tubes centrifuged at $14 \mathrm{~K}$ RCF for $15 \mathrm{~min}$. The upper (aqueous) phase was collected and DNA precipitated by addition of an equal volume of isopropanol (AR grade), mixing and centrifugation at $14 \mathrm{~K}$ RCF. The DNA pellet was washed in $70 \%$ ethanol and briefly air-dried before resuspension in between 50 and $200 \mu$ of a storage buffer (TE buffer; $10 \mathrm{mM}$ Tris, $1 \mathrm{mM}$ EDTA, PH 8). DNA was quantified using a Nanodrop 2000 instrument (Thermo, $\mathrm{NZ)}$.

\section{Polymerase chain reaction - restriction fragment length polymorphism (PCR-RFLP)}

The first enzyme reaction, to make copies of the DNA of interest (PCR), was performed i-Star Taq polymerase (Intron Ltd, USA) by initial strand separation at $95^{\circ} \mathrm{C}$ for 2 min followed by 35 repeats of temperature cycling between $95^{\circ} \mathrm{C} 30 \mathrm{~s}, 60^{\circ} \mathrm{C} 30 \mathrm{~s}, 72^{\circ} \mathrm{C} 30 \mathrm{~s}$, and a final extension step for $5 \mathrm{~min}$ at $72^{\circ} \mathrm{C}$. $100 \mathrm{ng}$ of DNA was used in each reaction. Gene annotations, SNP reference numbers, SNP codes used in this report, DNA primer sequences and details of restriction enzyme test are shown in Table 1. Reagents (DNA primers) and specific enzyme-based tests for the variants (namely the restriction fragment length polymorphism method) were designed by us using SNP Cutter (http://bioapp.psych.uic.edu/SNP_cutter.htm) or WatCut (http:// watcut.uwaterloo.ca/watcut/watcut/template.php) or taken from other published studies (as indicated in Table 1). The banding pattern from $10 \mu \mathrm{L}$ of digested DNA products separated on a gel in an electrical field by electrophoresis at $100 \mathrm{~V}$ for $45 \mathrm{~min}$ was used to determine which DNA variants were present in each patient sample. Ten percent of the samples were analysed twice as a quality control.

\section{Study design and SNP data quality controls}

A minimum sample size of 128 individuals was required to achieve a statistical power of 0.9 for individual SNPs; assuming a medium effect size of 0.5 , and a type I error rate of $<0.05$.

A test for suitability of the genetic tests (for Hardy Weinberg equilibrium) was carried out using an online calculator (http://oege. org/software/hardy-weinberg.html).

The measurement of pain intensity is complex. This is particularly evident in a plot of VRS against postsurgical PCA demands that shows many patients tolerating high VRS scores without increasing PCA usage (Figure 1). In this report we used statistical tests to measure potential influences of variants on VRS and PCA.

\section{Statistical Methods}

\section{SNP association analysis}

The VRS and PCA data were treated as ordinal. Briefly, for each SNP we analysed each genotype alone (i.e. the additive model, which 
Citation: Jacobson GM, Law CJ, Johnston H, Chaddock M, Kluger M, et al. (2014) The Utility of Single Nucleotide DNA Variations as Predictors of Postoperative Pain. J Anesth Clin Res 5: 401. doi:10.4172/2155-6148.1000401

Page 3 of 8

\begin{tabular}{|c|c|c|c|c|c|c|c|c|c|c|}
\hline Gene & Symbol & SNP & $\begin{array}{l}\text { Allele1/ } \\
\text { position }^{\alpha /} \\
\text { Allele2 }\end{array}$ & Type/change & $\begin{array}{l}\text { MAF } \\
(\%) \dagger\end{array}$ & PCR primers & $\begin{array}{l}\text { Size } \\
\text { (bp) }\end{array}$ & $\begin{array}{l}\text { Diagnostic } \\
\text { fragments } \\
\text { (bp) }\end{array}$ & Enzyme & Design \\
\hline \multirow[t]{2}{*}{$\begin{array}{l}\text { ATP-binding cassette, } \\
\text { sub-family B, member } 1\end{array}$} & \multirow{2}{*}{$A B C B 1$} & rs1045642 & С3435T & Synon. & $\mathrm{C} 43$ & $\begin{array}{l}\text { F AGCTGCTTGATGGCAAAGAAAT } \\
\text { R TGGTCGAACACTTTCATCCCTT }\end{array}$ & 433 & $\begin{array}{l}\text { T } 387 \\
\text { C } 236\end{array}$ & Mbol & $\ddagger$ \\
\hline & & rs 1128503 & $\mathrm{~T} 1236 \mathrm{C}$ & Synon. & T 44 & $\begin{array}{l}\text { F TCTTTGTCACTTTATCCAGC } \\
\text { R TCTCACCATCCССTCTGT }\end{array}$ & 502 & $\begin{array}{l}\text { C } 361 \\
\text { T } 416\end{array}$ & Haelll & $\S$ \\
\hline $\begin{array}{l}\text { Catechol-O- } \\
\text { methyltransferase }\end{array}$ & COMT & rs4680 & Val158Met & Non-synon. & A 39 & $\begin{array}{l}\text { F GTGGACGCCGTGATTCAGGAG } \\
\text { R CAGGCATGCACACCTTGTCCgTC }\end{array}$ & 199 & $\begin{array}{l}\text { A } 107 \\
\text { G } 83\end{array}$ & Tsp45I & $\ddagger$ \\
\hline $\begin{array}{c}\text { Cytochrome P450, } \\
\text { family } 3 \text {, subfamily A, } \\
\text { polypeptide } 4\end{array}$ & CYP $3 A 4$ & rs2242480 & C20230T & Intronic & T 33 & $\begin{array}{l}\text { F ACCCTGATGTCCAGCAGAAACT } \\
\text { RATAGAAAGCAGATGAACCAGAGCC }\end{array}$ & 284 & $\begin{array}{l}\text { C } 216 \\
\text { T } 284\end{array}$ & Rsal & ๆ \\
\hline $\begin{array}{c}\text { Cytochrome P450, } \\
\text { family } 3 \text {, subfamily A, } \\
\text { polypeptide } 5\end{array}$ & CYP $3 A 5$ & rs776746 & A6986G & Splice-3 & A 3 & $\begin{array}{l}\text { F GCATAGGAGATACCCACGTATCT } \\
\text { R TGGTCCAAACAGGGAAGAGgTA }\end{array}$ & 118 & $\begin{array}{l}\text { G } 97 \\
\text { A } 118\end{array}$ & Rsal & ** \\
\hline Neurobeachin1 & NBEA & rs7990537 & A11771G & Intronic & G 28 & $\begin{array}{l}\text { F CCCTTGGATGTTTGAACCTCTG } \\
\text { R TTTCCTAGATGCCCTTCACTGG }\end{array}$ & 433 & $\begin{array}{l}\text { A } 433 \\
\text { G } 332\end{array}$ & Bts1 & $\ddagger$ \\
\hline \multirow[t]{2}{*}{$\begin{array}{c}\text { Opioid } \\
\text { Receptor, mu } 1\end{array}$} & \multirow[t]{2}{*}{ OPRM1 } & rs1799971 & A118G & Non-synon. & G 19 & $\begin{array}{l}\text { F TCAACTTGTCCCACTTAGATCGC } \\
\text { R TGACCAGGAAGTTTCCGAAGAG }\end{array}$ & 180 & $\begin{array}{l}\text { A } 180 \\
\text { G } 158\end{array}$ & BstUI & $\ddagger$ \\
\hline & & rs2075572 & IVS2-691 & Intronic & G 40 & $\begin{array}{l}\text { F TAGCTCTGGTCAAGGCTAAAgAT } \\
\text { R CCCAGTACCAGGTTGGATGAGA }\end{array}$ & 154 & $\begin{array}{l}\text { G } 154 \\
\text { C } 134\end{array}$ & Mbol & $\ddagger$ \\
\hline \multirow[t]{2}{*}{$\begin{array}{l}\text { Potassium inwardly- } \\
\text { rectifying channel, } \\
\text { subfamily J, member } 6\end{array}$} & \multirow[t]{2}{*}{ KCNJ6 } & rs2070995 & A1032G & Synon. & A 20 & $\begin{array}{l}\text { F TAGAGGACCCCTCCTGGACT } \\
\text { R CGGAACATCAGGCACAGTTT }\end{array}$ & 298 & $\begin{array}{l}\text { A } 197 \\
\text { G } 175\end{array}$ & Mspl & $\ddagger$ \\
\hline & & rs6517442 & G-1250A & Upstream & G 24 & $\begin{array}{c}\text { F TGGCTATTCTTGTGCTGCTTT } \\
\text { R CAGTCATTTTTAGAGGGCAGtCA }\end{array}$ & 203 & $\begin{array}{l}\text { G } 203 \\
\text { A } 181\end{array}$ & Hincll & $\ddagger$ \\
\hline $\begin{array}{l}\text { Potassium voltage-gated } \\
\text { channel, delayed-rectifier, } \\
\text { subfamily } \mathrm{S}, \text { member } 1\end{array}$ & KCNS1 & rs734784 & Ile489Val & Non-synon. & G 40 & $\begin{array}{l}\text { F AGTTTGAGGACTTGCTGAGCcGC } \\
\text { R ATACATCTGAGGGTGTGGAGGC }\end{array}$ & 137 & $\begin{array}{l}\text { A } 137 \\
\text { G } 115\end{array}$ & BstUI & $\ddagger$ \\
\hline $\begin{array}{c}\text { Sodium channel, voltage- } \\
\text { gated, type IX, alpha } \\
\text { subunit }\end{array}$ & SCN9a & rs6746030 & Arg1150Trp & Non-synon. & A 11 & $\begin{array}{l}\text { F GTTTTCCTGATGTTCCACCAGATT } \\
\text { R TGAAGGAAGGCAGCATCCAAATTA }\end{array}$ & 244 & $\begin{array}{l}\text { A } 244 \\
\text { G } 172\end{array}$ & Rsal & $\ddagger$ \\
\hline
\end{tabular}

Lower case in primer sequences indicates bases changed to alter restriction sites. Key: Type codes with protein level consequence: Synon.-synonomous,- no amino acid change; Non-synon.- non-synonymous, amino acid change as indicated; Intronic - variant occurs in a non-coding portion of the gene; upstream - in region upstream (5' ) of the gene; Slice-3 - causes transcript splice variation in 3' of the gene; IVS2 -within intron 2 of the gene (full SNP code:IVS2-691 G>C); UTR-3' - 3' untranslated region. † Minor allele frequencies (MAF) are from http://www.ncbi.nlm.nih.gov/projects/SNP/. aChromosome positions are from HumRef sequence and transcript positions are from Genbank NM reference sequences. Primers and test design $\ddagger$ (us); §[44], \[45], **[46].

Table 1: SNPs examined in this study: allele frequency, PCR primer sequences and RFLP test details.
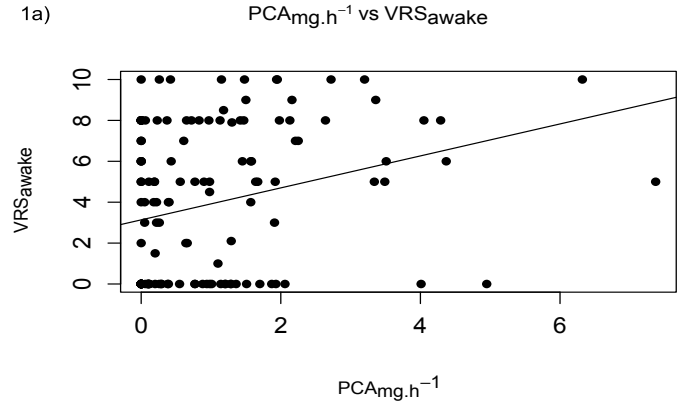

1b)

PCAmg.h ${ }^{-1}$ vs VRS $\mathrm{d} 1 \mathrm{~A}$

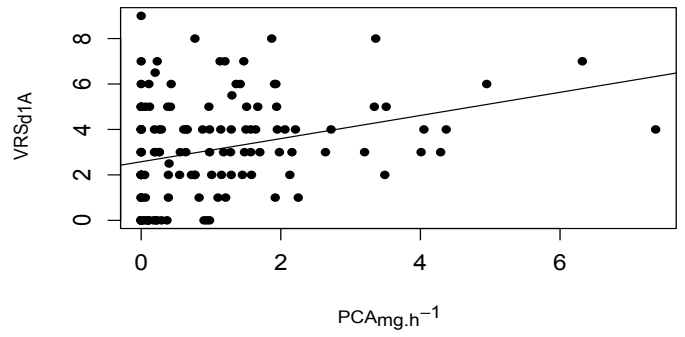

Figure 1: Scatterplots of VRS against PCA usage for all patients: A) VRS awake vs. PCA ${ }_{\text {mg.h }}{ }^{-1}$; and B) VRSd1A vs. PCAmg.h-1. Line of best fit is shown. Dots represent individual patients. 


\begin{tabular}{|c|c|c|c|c|c|}
\hline Basic information & & & & & \\
\hline Age & 43 yrs. $(33-55)$ & & & & \\
\hline $\begin{array}{l}\text { Gender } \\
\text { Height }\end{array}$ & $\begin{array}{c}105+/ 30 \AA \\
1.67 \mathrm{~m}(1.6-1.73)\end{array}$ & & & & \\
\hline BMI & $27.5(23.4-31.0)$ & & & & \\
\hline Caucasian & $107(79 \%)$ & & & & \\
\hline Polynesian & $18(13 \%)$ & & & & \\
\hline Asian & $10(8 \%)$ & & & & \\
\hline Surgery type & $\mathbf{n}$ & Duration (min) & $\begin{array}{c}\text { VRS }_{\text {awake }} \\
\text { median (IQR) }\end{array}$ & $\begin{array}{c}\text { VRS }_{\mathrm{d} 1 \mathrm{~A}} \\
\text { median (IQR) }\end{array}$ & $\mathrm{PCA}_{\mathrm{mg} \cdot \mathrm{h}-1}$ median (IQR) \\
\hline All & & $86(67-117)$ & $4(0-8)$ & $3(1-5)$ & $0.49(0-1.49)$ \\
\hline General & 54 & $86.5(72-127)$ & $0(0-5)$ & $2(0-5)$ & $0.39(0-1.2)$ \\
\hline Gynaecological & 65 & $83(54-113)$ & $6(3-5)$ & $3(2-4)$ & $0.98(0.12-1.9)$ \\
\hline Orthopaedic & 16 & $99.5(73-152)$ & $0(0-8)$ & $4(1-6)$ & $0.03(0-0.6)$ \\
\hline
\end{tabular}

$V_{R S}$ awake pain upon awakening; VRS ${ }_{d 1 A}$. VRS 20-24h postsurgery with light activity (e.g. coughing or movement); $\mathrm{PCA}_{\mathrm{mg} . \mathrm{h}-1}$ patient-controlled analgesia usage rate in mg. $\mathrm{h}^{-1}$; $\mathrm{BMI}$ - body mass index. Where appropriate values are expressed as median(interquartile range).

Table 2: Demographics, surgery and pain data $(n=135)$.

assumes that heterozygous individuals have a phenotype midway between the two homozygotes) and also grouped by the presence of either minor (less frequent) or major (more frequent) alleles (dominant or recessive models). For these models, where ' $d$ ' is the minor allele and ' $\mathrm{D}$ ' is the major allele, the recessive model compared values from individuals with ' $\mathrm{d} / \mathrm{d}$ ' against ' $\mathrm{D} / \mathrm{D}$ and $\mathrm{D} / \mathrm{d}$ ' genotypes and the dominant model compared values from individuals with ' $D / D$ ' against ' $D / d$ and $\mathrm{d} / \mathrm{d}$ ' genotypes. This approach avoids assumptions regarding mode of inheritance and limits the chance of missing potentially interesting associations and was similar to a method described previously [32,33]. Nonparametric statistical approaches were used including: 1) the 1-way ANOVA (with Kruskal-Wallis Z test) to examine the independent effect of all non-SNP variables on VRS or PCA, and 2) the general linear model (NCSS software) to measure associations between SNPs and VRS or PCA. For all tests a p-value $<0.05$ was considered statistically significant.

\section{Results}

A total of 135 patients from two hospitals in the central North Island of New Zealand (Waikato DHB and Northshore DHB) were recruited into the study. Demographic data, surgery type and duration, and median VRS data are presented in Table 2. No statistically significant associations were found between any pain score or PCA usage and either age, ethnicity or gender. However there was a strong association between VRS and surgery type $(\mathrm{p}<0.001)$. To incorporate the influence of surgery type it was included in all SNP association tests for all outcomes using the general linear model (up to 2-way). Hence $\mathrm{p}$ values reported here are for the SNP-outcome with consideration of surgery type.

Some samples $(<1 \%)$ failed to give a conclusive DNA test result and were excluded from further analysis. One NBEA variant (rs9565317) selected for inclusion in the study did not pass genetic testing (HardyWeinburg equilibrium) and was removed from the DNA variant panel. All results are expressed as median(interquartile range) unless otherwise stated.

\section{Association Analysis}

$V R S_{a v a c}$

No statistically significant SNP associations were found for VRS $V_{R S_{d 1 A}}$

An association between KCNJ6 A1032G variant and VRS $_{\text {d1A }}$ was found (Table 3). Patients with the $1032 \mathrm{G} / 1032 \mathrm{G}$ variant pair had higher median VRS scores when compared to patients with the 1032A/1032A or $1032 \mathrm{~A} / 1032 \mathrm{G}$ variant pairs $(\mathrm{p}=0.04$; dominant model) (Table 3). The difference in VRS score was only apparent when the actual surgery type was examined (Table 5) and this weak association showed a VRS range of between 2 and 3 for $1032 \mathrm{~A} / 1032 \mathrm{~A}$ or $1032 \mathrm{~A} / 1032 \mathrm{G}$ pairs and 2.3 to 5 for $1032 \mathrm{G} / 1032 \mathrm{G}$ pairs (Table 4 ). No significant associations were found for any other DNA variants and VRS ${ }_{\mathrm{d} 1 \mathrm{~A}}$ scores examined.

\section{Patient-controlled analgesia usage rate $\left(\mathrm{PCA}_{\mathrm{mg.h}}{ }^{-1}\right)$}

A significant association between PCA usage rate and $A B C B 1$ gene variant $\mathrm{C} 3435 \mathrm{~T}$ was found (Table 3 ). For all surgery types individuals with $3435 \mathrm{C} / 3435 \mathrm{C}$ or $3435 \mathrm{C} / 3435 \mathrm{~T}$ genotypes for C3435T consumed at a rate eight times faster in the same period - with median usage of $0.89(0.07-1.66)$ compared to just $0.11(0-0.52) \mathrm{mg} \cdot \mathrm{h}^{-1}$ in patients with the $3435 \mathrm{~T} / 3435 \mathrm{~T}$ genotype ( $<<0.03$; dominant model) (Table 5).

Also, for all surgery types combined, patients with the IVS2C/ IVS2C or IVS2C/IVS2G genotypes for the OPRM1 variant IVS2-691 had markedly higher median PCA usage rates of $0.77(0.01-1.56)$ compared to $0.24(0-1.26) \mathrm{mg} \cdot \mathrm{h}^{-1}$ in patients with the IVS2G/IVS2G variant pair (Table 5). This association was weakest in the gynaecological patient group where patients with the IVS2C/IVS2C and IVS2C/IVS2G genotype had just a $10 \%$ higher $\mathrm{PCA}_{\text {mg.h }}{ }^{-1}$ than those with the IVS2G/ IVS2G variant pair.

\section{Discussion}

The main findings of this study were that, in a broad-based sample of typical postoperative patients, a DNA variant (SNP A1032G in the KCNJ6 gene) was associated with $20-24 \mathrm{~h}$ postoperative pain and two DNA variants (SNPs C3435T in ABCB1 and IVS2-691 in OPRM1) were associated with a higher PCA usage rate. As noted above, surgery type had a strong influence on VRS - this was statistically significant for $\mathrm{VRS}_{\text {awake }}$ - and surgery type was included in our statistical models.

We found that patients with two copies of the $1032 \mathrm{G}$ variant (i.e. 1032G/1032G) in KCNJ6 had higher $\mathrm{VRS}_{\mathrm{d1A}}$ when compared to individuals with the variant pairs $1032 \mathrm{G} / 1032 \mathrm{~A}$ or $1032 \mathrm{~A} / 1032 \mathrm{~A}$ but this was only evident with consideration of the individual surgery types. Nishizawa [27] did not see any association between this variant and pain score but reported an association between the 1032A DNA variant and increased postoperative rescue analgesia requirements. They explain their result by stating that the alternative $1032 \mathrm{G}$ variant 
Citation: Jacobson GM, Law CJ, Johnston H, Chaddock M, Kluger M, et al. (2014) The Utility of Single Nucleotide DNA Variations as Predictors of Postoperative Pain. J Anesth Clin Res 5: 401. doi:10.4172/2155-6148.1000401

Page 5 of 8

\begin{tabular}{|c|c|c|c|c|c|c|c|c|c|c|c|c|c|c|c|c|}
\hline \multirow[b]{2}{*}{ Gene } & \multirow[b]{2}{*}{ SNP } & \multirow[b]{2}{*}{ Genotype } & \multirow[b]{2}{*}{ Count } & \multirow[b]{2}{*}{$\%$} & \multirow{2}{*}{\begin{tabular}{|c|} 
VRS $_{\text {awake }}$ \\
(IQR) \\
\end{tabular}} & \multicolumn{3}{|c|}{$p$ values } & \multirow{2}{*}{$\begin{array}{l}\text { VRS }_{\mathrm{d} 1 \mathrm{~A}} \\
\text { (IQR) }\end{array}$} & \multicolumn{3}{|c|}{$p$ values } & \multirow{2}{*}{$\begin{array}{c}\text { PCA }_{\text {mg.h }}{ }^{-1} \\
\text { (IQR) }\end{array}$} & \multicolumn{3}{|c|}{ p values } \\
\hline & & & & & & Addit. & Dom. & Rec. & & Addit. & Dom. & Rec. & & Addit. & Dom. & Rec. \\
\hline \multirow[t]{6}{*}{ ABCB1 } & \multirow[t]{3}{*}{ C3435T } & $\mathrm{C} / \mathrm{C}$ & 37 & 27.2 & $4(0-8)$ & \multirow[t]{3}{*}{0.76} & \multirow[t]{3}{*}{0.63} & \multirow[t]{3}{*}{0.81} & $3(1-5)$ & \multirow[t]{3}{*}{0.35} & \multirow[t]{3}{*}{0.23} & \multirow[t]{3}{*}{0.25} & $0.77(0.2-1.5)$ & \multirow[t]{3}{*}{0.09} & \multirow[t]{3}{*}{0.03} & \multirow[t]{3}{*}{0.23} \\
\hline & & $\mathrm{C} / \mathrm{T}$ & 63 & 46.3 & $5(0-8)$ & & & & $3(1-5)$ & & & & $0.9(1-1.9)$ & & & \\
\hline & & $T / T$ & 36 & 26.5 & $4(0-8)$ & & & & $3(0.8-4)$ & & & & $0.11(0-0.52)$ & & & \\
\hline & T1236C & $T / T$ & 28 & 22.6 & $4(0-8)$ & 0.35 & 0.67 & 0.28 & $3(5)$ & 0.22 & 0.09 & 0.56 & $0.88(0.11-2)$ & 0.8 & 0.7 & 0.78 \\
\hline & & $\mathrm{C} / \mathrm{T}$ & 62 & 50.0 & $5(0-8)$ & & & & $3(1-5)$ & & & & $0.69(0-1.46)$ & & & \\
\hline & & $\mathrm{C} / \mathrm{C}$ & 34 & 27.4 & $4(0-8)$ & & & & $4(2-6)$ & & & & $0.23(0-1.23)$ & & & \\
\hline СOMT & Val158Met & G/G & 34 & 25.4 & $5(0-8)$ & 0.35 & 0.51 & 0.42 & $3(1-3.3)$ & 0.97 & 0.89 & 0.92 & $0.59(0.12-2.1)$ & 0.15 & 0.72 & 0.05 \\
\hline & & $\mathrm{G} / \mathrm{A}$ & 48 & 35.8 & $3(0-6)$ & & & & $3(2-5)$ & & & & $0.66(0-1.3)$ & & & \\
\hline & & $\mathrm{A} / \mathrm{A}$ & 52 & 38.8 & $5(0-8)$ & & & & $4(1-4.5)$ & & & & $0.34(0-1.66)$ & & & \\
\hline CYP3A4 & C20230T & $\mathrm{A} / \mathrm{A}$ & 9 & 6.6 & $5(0-7)$ & 0.17 & 0.88 & 0.26 & $2(0-3.5)$ & 0.78 & 0.91 & 0.61 & $0.26(0-1.5)$ & 0.92 & 0.61 & 0.99 \\
\hline & & $A / G$ & 38 & 27.7 & $3(0-6)$ & & & & $4(2-5)$ & & & & $0.715(0-1.5)$ & & & \\
\hline & & G/G & 90 & 65.7 & $4(0-8)$ & & & & $3(1-5)$ & & & & $0.55(0.06-1.54)$ & & & \\
\hline CYP3A5 & A6986G & $\mathrm{A} / \mathrm{A}$ & 5 & 3.7 & $0(0-3)$ & 0.57 & 0.18 & 0.32 & $0(0-2)$ & 0.25 & 0.23 & 0.06 & $0(0-1.32)$ & 0.33 & 0.24 & 0.38 \\
\hline & & $\mathrm{G} / \mathrm{A}$ & 26 & 19.1 & $0(0-6)$ & & & & $2(1-4.5)$ & & & & $0.09(0-1.32)$ & & & \\
\hline & & G/G & 105 & 77.2 & $5(0-8)$ & & & & $3(2-5)$ & & & & $0.66(0.1-1.57)$ & & & \\
\hline NBEA & A11771G & G/G & 1 & 0.9 & 5 & 0.22 & 0.72 & 0.43 & 1 & 0.93 & 0.88 & 0.46 & 0.39 & 0.73 & 0.46 & 0.75 \\
\hline & & $\mathrm{G} / \mathrm{A}$ & 27 & 24.3 & $4(0-8)$ & & & & $3(1-5.5)$ & & & & $0.38(0-1.36)$ & & & \\
\hline & & $\mathrm{A} / \mathrm{A}$ & 83 & 74.8 & $5(0-8)$ & & & & $3(2-3)$ & & & & $0.55(0.1-1.45)$ & & & \\
\hline OPRM1 & A118G & G/G & 3 & 2.2 & $0(0-8)$ & 0.85 & 0.69 & 0.95 & $3(2-4)$ & 0.16 & 0.09 & 0.83 & $0.98(0.37-1.2)$ & 0.62 & 0.37 & 0.95 \\
\hline & & $A / G$ & 31 & 22.6 & $5(0-8)$ & & & & $3(1-5)$ & & & & $0.77(0-1.64)$ & & & \\
\hline & & $\mathrm{A} / \mathrm{A}$ & 103 & 75.2 & $4(0-7)$ & & & & $3(1-5)$ & & & & $0.49(0-1.45)$ & & & \\
\hline & IVS2-691 & G/G & 40 & 29.2 & $2(0-5)$ & 0.11 & 0.07 & 0.09 & $2(0-4)$ & 0.68 & 0.74 & 0.35 & $0.24(0-1.26)$ & 0.15 & 0.63 & 0.04 \\
\hline & & $\mathrm{G} / \mathrm{C}$ & 59 & 43.1 & $5(0-8)$ & & & & $4(2-5)$ & & & & $0.72(0-1.87)$ & & & \\
\hline & & $\mathrm{C} / \mathrm{C}$ & 38 & 27.7 & $5(0-8)$ & & & & $3(1-4)$ & & & & $0.77(0.06-1.45)$ & & & \\
\hline KCNJ6 & A1032G & $\mathrm{A} / \mathrm{A}$ & 14 & 9.9 & $4(0-6)$ & 0.78 & 0.66 & 0.5 & $3(1-5)$ & 0.13 & 0.04 & 0.97 & $0.23(0-1.59)$ & 0.5 & 0.51 & 0.58 \\
\hline & & $\mathrm{A} / \mathrm{G}$ & 58 & 41.1 & $4(0-8)$ & & & & $3(1-4)$ & & & & $0.6(0-1.53)$ & & & \\
\hline & & G/G & 69 & 48.9 & $5(0-8)$ & & & & $3(2-5)$ & & & & $0.52(0.18-1.44)$ & & & \\
\hline & G-1250A & G/G & 20 & 14.6 & $5(0-8)$ & 0.2 & 0.07 & 0.55 & $1(0-4)$ & 0.1 & 0.27 & 0.2 & $0.1(0-1)$ & 0.49 & 0.59 & 0.27 \\
\hline & & $\mathrm{G} / \mathrm{A}$ & 67 & 48.9 & $5(0-8)$ & & & & $3(2-5)$ & & & & $0.8(0.12-1.57)$ & & & \\
\hline & & $\mathrm{A} / \mathrm{A}$ & 50 & 36.5 & $3(0-7)$ & & & & $3(1-5)$ & & & & $0.38(0-1.5)$ & & & \\
\hline KCNS1 & Ile489Val & G/G & 24 & 18.8 & $5(0-8)$ & 0.54 & 0.29 & 0.58 & $3(1-4)$ & 0.22 & 0.25 & 0.38 & $0.58(0.12-1.53)$ & 0.29 & 0.28 & 0.49 \\
\hline & & $A / G$ & 58 & 45.3 & $5(0-8)$ & & & & $3(2-4)$ & & & & $0.69(0.05-1.65)$ & & & \\
\hline & & $\mathrm{A} / \mathrm{A}$ & 46 & 35.9 & $3(0-6)$ & & & & $3(1-5)$ & & & & $0.22(0-1.22)$ & & & \\
\hline SCN9a & Arg1150Trp & $\mathrm{A} / \mathrm{A}$ & 0 & 0.0 & $\mathrm{n} / \mathrm{a}$ & 0.37 & 0.37 & $\mathrm{n} / \mathrm{a}$ & $\mathrm{n} / \mathrm{a}$ & 0.28 & 0.28 & $\mathrm{n} / \mathrm{a}$ & $\mathrm{n} / \mathrm{a}$ & 0.06 & 0.06 & $\mathrm{n} / \mathrm{a}$ \\
\hline & & $\mathrm{G} / \mathrm{A}$ & 32 & 23.4 & $4(0-7)$ & & & & $3(1-5)$ & & & & $0.11(0-0.9)$ & & & \\
\hline & & G/G & 105 & 76.6 & $4(0-8)$ & & & & $3(1-4)$ & & & & $0.77(0.1-1.56)$ & & & \\
\hline
\end{tabular}

Shaded cells with $\mathrm{p}$ values in italics, bolded and underlined indicate that a significant association ( $\mathrm{p} \leq 0.05$ ) was detected. Addit. - additive model; Dom. - dominant model; Rec. - recessive model; IRQ - interquartile range.

Table 3: SNP frequencies, genotyping data and results of association analysis for all study subjects.

seems to confer an improved efficiency in mediating the transmission of opioid signaling compared with the 1032A variant. Another study found association between eight $K C N J 6$ variants and postoperative oral analgesia usage but pain scores were not tested (nor were the A1032G or G-1250A variants) [34]. The exact function of these genetically synonymous KCNJ6 variants is currently unknown but others suggest that the $\mathrm{A} 1032 \mathrm{G}$ variant may regulate gene usage levels via mRNA secondary structure, with lower gene expression in individuals with the 1032A allele [27].

The association we describe between the $A B C B 1$ variant $3435 \mathrm{~T}$ and lower PCA usage rate reflects the findings of another recent study that reports lower PCA requirements in individuals homozygous for the $3435 \mathrm{~T}$ variant [5]. This effect is very likely due to the higher expression of the efflux pump that is driven from the $3435 \mathrm{C}$ variant $[35,36]$. Individuals carrying this allele therefore have a stronger barrier to opioids at the blood brain barrier so that less of the drug is available at receptors in the CNS [37] and a relatively higher PCA dose is required.
For the OPRM1 variant IVS2-691, patients with IS2C/IS2C or IS2C/ IS2G variant pairs had median $\mathrm{PCA}_{\mathrm{mg} . \mathrm{h}^{-1}}$ pain levels more than two-fold higher than those with the IS2G/IS2G variant pairs across all surgery types. We could not find other studies linking the variant to variation in either postoperative pain or PCA requirements. However, the variant has been studied for effects on oral morphine efficacy in cancer patients although no association was found [38] and another study reported no association between the variant and morphine pharmacodynamics using plasma samples from healthy volunteers [39]. The IVS2-691 variant may exert the observed effects by causing variation in slicing patterns of mRNA transcripts or may be simply 'tagging' a nearby variant that actually confers some functional effect and is simply inherited during meiosis with the IVS2-691 variant due to proximity on the same chromosome.

\section{Possible clinical applicability}

In our patient cohort different DNA variants showed different associations for VRS (i.e. with KCNJ6 gene variant) and PCA (i.e. 
Citation: Jacobson GM, Law CJ, Johnston H, Chaddock M, Kluger M, et al. (2014) The Utility of Single Nucleotide DNA Variations as Predictors of Postoperative Pain. J Anesth Clin Res 5: 401. doi:10.4172/2155-6148.1000401

Page 6 of 8

\begin{tabular}{|c|c|c|c|c|c|c|c|}
\hline Gene & SNP & Model & Surgery & Groups & VRS (IRQ) & Count & $p$ value \\
\hline KCNJ6 & A1032G & Dom. & All & $\begin{array}{c}1032 A / A \text { and } 1032 A / G \\
1032 G / G\end{array}$ & $\begin{array}{l}3(1-4) \\
3(2-5)\end{array}$ & $\begin{array}{l}64 \\
67\end{array}$ & 0.18 \\
\hline & & & $\begin{array}{c}\text { General } \\
\text { Gynaecological } \\
\text { Orthopaedic }\end{array}$ & $\begin{array}{c}1032 \mathrm{~A} / \mathrm{A} \text { and } 1032 \mathrm{~A} / \mathrm{G} \\
1032 \mathrm{G} / \mathrm{G} \\
1032 \mathrm{~A} / \mathrm{A} \text { and } 1032 \mathrm{~A} / \mathrm{G} \\
1032 \mathrm{G} / \mathrm{G} \\
1032 \mathrm{~A} / \mathrm{A} \text { and } 1032 \mathrm{~A} / \mathrm{G} \\
1032 \mathrm{G} / \mathrm{G}\end{array}$ & $\begin{array}{c}2(0-5) \\
2.3(1-4.3) \\
3(2-4) \\
4(2-5) \\
3(0.5-4) \\
5(4-6)\end{array}$ & $\begin{array}{c}26 \\
26 \\
29 \\
34 \\
9 \\
7\end{array}$ & $\underline{0.04}$ \\
\hline
\end{tabular}

Dom. - dominant model; Rec. - recessive model. Underlined p value is for general linear model with 'surgery type' included.

Table 4: Details of significant association found between SNPS and VRS $\mathrm{d} 1 \mathrm{~A}$

\begin{tabular}{|c|c|c|c|c|c|c|c|}
\hline Gene & SNP & Model & Surgery & Groups & VRS (IRQ) & Count & $p$ value \\
\hline ABCB1 & C3435T & Dom. & All & $\begin{array}{c}3435 \mathrm{C} / \mathrm{C} \text { or } 3435 \mathrm{C} / \mathrm{T} \\
3435 \mathrm{~T} / 3435 \mathrm{~T}\end{array}$ & $\begin{array}{c}0.89(0.07-1.66) \\
0.11(0-0.52)\end{array}$ & $\begin{array}{c}100 \\
36\end{array}$ & 0.02 \\
\hline & & & $\begin{array}{l}\text { General } \\
\text { Gynae. } \\
\text { Ortho }\end{array}$ & $\begin{array}{c}3435 \mathrm{C} / \mathrm{C} \text { or } 3435 \mathrm{C} / \mathrm{T} \\
3435 \mathrm{~T} / 3435 \mathrm{~T} \\
3435 \mathrm{C} / \mathrm{C} \text { or } 3435 \mathrm{C} / \mathrm{T} \\
3435 \mathrm{~T} / 3435 \mathrm{~T} \\
3435 \mathrm{C} / \mathrm{C} \text { or } 3435 \mathrm{C} / \mathrm{T} \\
3435 \mathrm{~T} / 3435 \mathrm{~T}\end{array}$ & $\begin{array}{c}0.77(0.12-1.29) \\
0.09(0-0.39) \\
1.22(0.19-1.94) \\
0.26(0-0.21) \\
0.06(0-1.42) \\
0(0-0.11)\end{array}$ & $\begin{array}{l}41 \\
13 \\
48 \\
17 \\
11 \\
5\end{array}$ & $\underline{0.03}$ \\
\hline OPRM1 & IVS2-691 & Rec. & All & $\begin{array}{l}\text { IVS2C/ IVS2G or IVS2C/ IVS2C } \\
\text { IVS2G/ IVS2G }\end{array}$ & $\begin{array}{c}0.77(0.01-1.56) \\
0.24(0-1.26)\end{array}$ & $\begin{array}{l}96 \\
40\end{array}$ & 0.03 \\
\hline & & & $\begin{array}{l}\text { General } \\
\text { Gynae. } \\
\text { Ortho. }\end{array}$ & $\begin{array}{l}\text { IVS2C/ IVS2C or IVS2C/ IVS2G } \\
\text { IVS2G/ IVS2G } \\
\text { IVS2C/ IVS2C or IVS2C/ IVS2G } \\
\text { IVS2G/ IVS2G } \\
\text { IVS2C/ IVS2C or IVS2C/ IVS2G } \\
\text { IVS2G/ IVS2G }\end{array}$ & $\begin{array}{c}0.69(0.03-1.26) \\
0.24(0-1.05) \\
0.98(0.13-1.81) \\
0.89(0.08-1.92) \\
0.06(0-1.42) \\
0(0-105)\end{array}$ & $\begin{array}{l}36 \\
18 \\
49 \\
16 \\
11 \\
5\end{array}$ & $\underline{0.04}$ \\
\hline
\end{tabular}

Dom. - dominant model; Rec. - recessive model. IQR - interquartile range. Underlined p value is for general linear model with 'surgery type' included.

Table 5: Significant associations found between SNPs and PCAmg. $\mathrm{h}^{-1}$ with dominant or recessive models

with $A B C B 1$ and $O R P M 1$ variants). For VRS, the association with the KCNJ6 variant was weak and is unlikely to have much clinical utility. PCA usage is a composite endpoint that involves a complex relationship including genetic effects acting on the intensity of the pain experienced by the patient and pharmacogenetic effects on the perceived potency of the morphine. Whether preoperative knowledge of genotypes that associated with PCA, for example the 3435T/3435T variant pair for the $A B C B 1$ gene (which had a $>8$-fold lower PCA usage rate and was found in around $26 \%$ of all study subjects), would allow the avoidance of postoperative PCA prescription is as yet untested. Such possible therapeutic interventions require formal testing in prospective studies. However, some groups have noted obstacles to adoption of this type of pharmacogenetic testing, including the unreliability of some of the evidence for associations due to weaknesses in the design of some candidate gene association studies and/or their irreproducibility [40]. Overlying these issues is the confounding factor of the subjective nature of pain perception [41].

\section{Questions about the neuroscience of postoperative pain}

While the $A B C B 13435 \mathrm{C}$ variant association with higher PCA usage rate for certain types of surgery agree with previous studies, the association for the KCNJ6 A1032G variant with VRS was unexpected in light of evidence reported by Nishizawa [27]. Although it is possible that some associations we found evidence for may be statistical artefacts, we believe that our results call into question the simplistic pharmacokinetic and pharmacodynamic explanations of the genetic influences on pain and highlights the paucity of our knowledge of the interactions between nociceptive and pharmacological mechanisms.
One notable finding in our study was the lack of any association between the OPRM1 A118G variant and any of the clinical outcomes. This may be due to a masking of the association due to the low percentage of Asian patients in the study (around 8\%). Around 15\% of the Asian population are minor allele homozygous for the variant (i.e. have the variant pair $118 \mathrm{G} / 118 \mathrm{G}$ ) compared to $<2 \%$ in the Caucasian population. However, other studies have also failed to detect any association with the A118G variant and postoperative pain (VRS). Janicki [42] did not find any association for 118G with either pain score or morphine requirements following surgery and De Gregori [43] did not detect any association with PCA in a similar study (they did not examine pain score data).

\section{Conclusion}

We found evidence for various associations between DNA variants in $A B C B 1, O P R M 1$, and KCNJ6 and postoperative pain and/or PCA usage. However, these results would appear to be more of scientific than clinical interest because the strength of the correlations do not appear to be strong enough to form the basis for useful clinical prediction.

Importantly, we found no pain association for examined variants in COMT, CYP3A4, CYP3A5, KCNS1, NBEA and SCN9a genes. This suggests that the particular variants studied in these genes are unlikely to have value in prediction of postoperative pain in a typical population of elective surgery patients. Future studies should focus on defining a more useful panel of DNA variants for reliable prediction of postoperative pain. 
Citation: Jacobson GM, Law CJ, Johnston H, Chaddock M, Kluger M, et al. (2014) The Utility of Single Nucleotide DNA Variations as Predictors of Postoperative Pain. J Anesth Clin Res 5: 401. doi:10.4172/2155-6148.1000401

\section{Acknowledgement}

We wish to thank Dr Pawel Olszewski for suggestion of inclusion of NBEA variants and Ben Cardwell and Olivia Patty for technical assistance. This study was supported by the Australian and New Zealand College of Anaesthetists grant 09/020 (GMJ, CJL) and by the University of Waikato (GMJ).

\section{Authors contributions}

GJ selected relevant genes for the study, coordinated sample collection and processing, designed and carried out the RFLP tests and drafted the manuscript. $\mathrm{CL}$ assisted in study design, applied for ethics committee approval, recruited Waikato DHB patients, collated drug usage information, interviewed patients for relevant pain score information and helped to draft the manuscript. $\mathrm{HJ}$ helped design some RFLP tests, extracted DNA from patient samples and performed genotyping for some variants. MC and MK collected and collated Northshore DHB samples, assisted in study design and commented on the manuscript. RC participated in the design of the study and in data analysis. JS had the initial idea for the study, participated in its design and coordination and helped to draft the manuscript. All authors read and approved the final manuscript.

\section{References}

1. Tracey I (2008) Imaging pain. Br J Anaesth 101: 32-39.

2. Kim H, Ramsay E, Lee H, Wahl S, Dionne RA (2009) Genome-wide association study of acute post-surgical pain in humans. Pharmacogenomics 10: 171-179.

3. Ikeda K, Ide S, Han W, Hayashida M, Uhl GR, et al. (2005) How individual sensitivity to opiates can be predicted by gene analyses. Trends Pharmacol Sci 26: 311-317.

4. Wandel C, Kim R, Wood M, Wood A (2002) Interaction of morphine, fentanyl, sufentanil, alfentanil, and loperamide with the efflux drug transporter P-glycoprotein. Anesthesiology 96: 913-920.

5. Candiotti K, Yang Z, Xue L, Zhang Y, Rodriguez Y, et al. (2013) Singlenucleotide polymorphism C3435T in the ABCB1 gene is associated with opioid consumption in postoperative pain. Pain Med 14: 1977-1984.

6. Levran O, O'Hara K, Peles E, Li D, Barral S, et al. (2008) ABCB1 (MDR1) genetic variants are associated with methadone doses required for effective treatment of heroin dependence. Hum Mol Genet 17: 2219-2227.

7. Andersen S, Skorpen F (2009) Variation in the COMT gene: implications for pain perception and pain treatment. Pharmacogenomics 10: 669-684.

8. Scanlon PD, Raymond FA, Weinshilboum RM (1979) Catechol-O methyltransferase: thermolabile enzyme in erythrocytes of subjects homozygous for allele for low activity. Science 203: 63-65.

9. Reyes-Gibby CC, Shete S, Rakvåg T, Bhat SV, Skorpen F, et al. (2007) Exploring joint effects of genes and the clinical efficacy of morphine for cancer pain: OPRM1 and COMT gene. Pain 130: 25-30.

10. Ahlers SJ, Elens LL, van Gulik L, van Schaik RH, van Dongen EP, et al. (2013) The Val158Met polymorphism of the COMT gene is associated with increased pain sensitivity in morphine-treated patients undergoing a painful procedure after cardiac surgery. Br J Clin Pharmacol 75: 1506-1515.

11. Jin M, Gock SB, Jannetto PJ, Jentzen JM, Wong SH (2005) Pharmacogenomics as molecular autopsy for forensic toxicology: genotyping cytochrome P450 $3 \mathrm{~A} 4{ }^{*} 1 \mathrm{~B}$ and $3 \mathrm{~A} 5{ }^{\star} 3$ for 25 fentanyl cases. J Anal Toxicol 29: 590-598.

12. Dai D, Tang J, Rose R, Hodgson E, Bienstock RJ, et al. (2001) Identification of variants of CYP3A4 and characterization of their abilities to metabolize testosterone and chlorpyrifos. J Pharmacol Exp Ther 299: 825-831.

13. Zhang W, Chang YZ, Kan QC, Zhang LR, Li ZS, et al. (2010) CYP3A4*1G genetic polymorphism influences CYP3A activity and response to fentanyl in Chinese gynecologic patients. Eur J Clin Pharmacol 66: 61-66.

14. Bosch TM, Meijerman I, Beijnen JH, Schellens JH (2006) Genetic polymorphisms of drug-metabolising enzymes and drug transporters in the chemotherapeutic treatment of cancer. Clin Pharmacokinet 45: 253-285.

15. Kuehl P, Zhang J, Lin Y, Lamba J, Assem M, et al. (2001) Sequence diversity in CYP3A promoters and characterization of the genetic basis of polymorphic CYP3A5 expression. Nat Genet 27: 383-391.

16. Niesmann K, Breuer D, Brockhaus J, Born G, Wolff I, et al. (2011) Dendritic spine formation and synaptic function require neurobeachin. Nat Commun 2: 557

17. Olszewski PK, Rozman J, Jacobsson JA, Rathkolb B, Strömberg S, et al. (2012)
Neurobeachin, a regulator of synaptic protein targeting, is associated with body fat mass and feeding behavior in mice and body-mass index in humans. PLoS Genet 8: e1002568.

18. Pasternak GW (2001) Insights into mu opioid pharmacology the role of mu opioid receptor subtypes. Life Sci 68: 2213-2219.

19. Pasternak GW (2001) The pharmacology of mu analgesics: from patients to genes. Neuroscientist 7: 220-231.

20. Oertel BG, Kettner M, Scholich K, Renne C, Roskam B, et al. (2009) A common human micro-opioid receptor genetic variant diminishes the receptor signaling efficacy in brain regions processing the sensory information of pain. $J$ Biol Chem 284: 6530-6535.

21. Sia AT, Lim Y, Lim EC, Goh RW, Law HY, et al. (2008) A118G single nucleotide polymorphism of human mu-opioid receptor gene influences pain perception and patient-controlled intravenous morphine consumption after intrathecal morphine for postcesarean analgesia. Anesthesiology 109: 520-526.

22. Sia AT, Lim Y, Lim EC, Ocampo CE, Lim WY, et al. (2013) Influence of muopioid receptor variant on morphine use and self-rated pain following abdominal hysterectomy. J Pain 14: 1045-1052.

23. Chou WY, Yang LC, Lu HF, Ko JY, Wang CH, et al. (2006) Association of mu-opioid receptor gene polymorphism (A118G) with variations in morphine consumption for analgesia after total knee arthroplasty. Acta Anaesthesiol Scand 50: 787-792.

24. Tan EC, Lim EC, Teo YY, Lim Y, Law HY, et al. (2009) Ethnicity and OPRM variant independently predict pain perception and patient-controlled analgesia usage for post-operative pain. Mol Pain 5: 32.

25. Shabalina SA, Zaykin DV, Gris P, Ogurtsov AY, Gauthier J, et al. (2009) Expansion of the human mu-opioid receptor gene architecture: novel functional variants. Hum Mol Genet 18: 1037-1051.

26. Ikeda K, Kobayashi T, Kumanishi T, Niki H, Yano R (2000) Involvement of G-protein-activated inwardly rectifying K (GIRK) channels in opioid-induced analgesia. Neurosci Res 38: 113-116.

27. Nishizawa D, Nagashima M, Katoh R, Satoh Y, Tagami M, et al. (2009) Association between KCNJ6 (GIRK2) gene polymorphisms and postoperative analgesic requirements after major abdominal surgery. PLoS One 4: e7060.

28. Sahni N, Anand LK, Gombar K, Gombar S (2012) Effect of intraoperative depth of anesthesia on postoperative pain and analgesic requirement: $A$ randomized prospective observer blinded study. J Anaesthesiol Clin Pharmacol 28: 266267.

29. Mitsis GD, lannetti GD, Smart TS, Tracey I, Wise RG (2008) Regions of interest analysis in pharmacological fMRI: how do the definition criteria influence the inferred result? Neuroimage 40: 121-132.

30. Estacion M, Harty TP, Choi JS, Tyrrell L, Dib-Haij SD, et al. (2009) A sodium channel gene SCN9A polymorphism that increases nociceptor excitability. Ann Neurol 66: 862-866.

31. Reimann F, Cox JJ, Belfer I, Diatchenko L, Zaykin DV, et al (2010) Pain perception is altered by a nucleotide polymorphism in SCN9A. Proc Natl Acad Sci U S A 107: 5148-5153.

32. Fukuda K, Hayashida M, Ide S, Saita N, Kokita Y, et al. (2009) Association between OPRM1 gene polymorphisms and fentanyl sensitivity in patients undergoing painful cosmetic surgery. Pain 147: 194-201.

33. Kolesnikov Y, Gabovits B, Levin A, Voiko E, Veske A (2011) Combined catechol O-methyltransferase and mu-opioid receptor gene polymorphisms affect morphine postoperative analgesia and central side effects. Anesth Analg 112 448-453.

34. Bruehl S, Denton JS, Lonergan D, Koran ME, Chont M, et al. (2013) Associations between KCNJ6 (GIRK2) gene polymorphisms and pain-related phenotypes. Pain 154: 2853-2859.

35. Hoffmeyer S, Burk O, von Richter O, Arnold HP, Brockmoller J, et al. (2000) Functional polymorphisms of the human multidrug-resistance gene: multiple sequence variations and correlation of one allele with P-glycoprotein expression and activity in vivo. Proc Natl Acad Sci USA 97: 3473-3478.

36. Kim RB, Leake BF, Choo EF, Dresser GK, Kubba SV, et al. (2001) Identification of functionally variant MDR1 alleles among European Americans and African Americans. Clin Pharmacol Ther 70: 189-199. 
Citation: Jacobson GM, Law CJ, Johnston H, Chaddock M, Kluger M, et al. (2014) The Utility of Single Nucleotide DNA Variations as Predictors of Postoperative Pain. J Anesth Clin Res 5: 401. doi:10.4172/2155-6148.1000401

Page 8 of 8

37. Lotsch J, Skarke C, Tegeder I, Geisslinger G (2002) Drug interactions with patient-controlled analgesia. Clin Pharmacokinet 41: 31-57.

38. Klepstad P, Rakvag TT, Kaasa S, Holthe M, Dale O, et al. (2004) The 118 $A>G$ polymorphism in the human mu-opioid receptor gene may increase morphine requirements in patients with pain caused by malignant disease. Acta Anaesthesiol Scand 48: 1232-1239.

39. Lötsch J, Geisslinger G (2006) Relevance of frequent mu-opioid receptor polymorphisms for opioid activity in healthy volunteers. Pharmacogenomics $J$ 6: 200-210.

40. Muralidharan A, Smith MT (2011) Pain, analgesia and genetics. J Pharm Pharmacol 63: 1387-1400.

41. Vuilleumier PH, Stamer UM, Landau R (2012) Pharmacogenomic considerations in opioid analgesia. Pharmgenomics Pers Med 5: 73-87.

42. Janicki PK, Schuler G, Francis D, Bohr A, Gordin V, et al. (2006) A genetic association study of the functional $\mathrm{A} 118 \mathrm{G}$ polymorphism of the human muopioid receptor gene in patients with acute and chronic pain. Anesth Analg 103: 1011-1017.
43. De Gregori M, Garbin G, De Gregori S, Minella CE, Bugada D, et al. (2013) Genetic variability at COMT but not at OPRM1 and UGT2B7 loci modulates morphine analgesic response in acute postoperative pain. European journal of clinical pharmacology 69: 1651-1658.

44. Kato M, Fukuda T, Serretti A, Wakeno M, Okugawa G, et al. (2008) ABCB1 (MDR1) gene polymorphisms are associated with the clinical response to paroxetine in patients with major depressive disorder. Prog Neuropsychopharmacol Biol Psychiatry 32: 398-404.

45. Gao Y, Zhang LR, Fu Q (2008) CYP3A4*1G polymorphism is associated with lipid-lowering efficacy of atorvastatin but not of simvastatin. Eur J Clin Pharmacol 64: 877-882.

46. Willrich MA, Hirata MH, Genvigir FD, Arazi SS, Rebecchi IM, et al. (2008) CYP3A53A allele is associated with reduced lowering-lipid response to atorvastatin in individuals with hypercholesterolemia. Clin Chim Acta 398: 1520.
Citation: Jacobson GM, Law CJ, Johnston H, Chaddock M, Kluger M, et al. (2014) The Utility of Single Nucleotide DNA Variations as Predictors of Postoperative Pain. J Anesth Clin Res 5: 401. doi:10.4172/2155-6148.1000401
Submit your next manuscript and get advantages of OMICS Group submissions

Unique features:

- User friendly/feasible website-translation of your paper to 50 world's leading languages

Audio Version of published paper

Digital articles to share and explore

Special features:

350 Open Access Journals

30,000 editorial team

21 days rapid review process

Quality and quick editorial, review and publication processing

Indexing at PubMed (partial), Scopus, EBSCO, Index Copernicus and Google Scholar etc

Sharing Option: Social Networking Enabled

- Authors, Reviewers and Editors rewarded with online Scientific Credits

Better discount for your subsequent articles

Submit your manuscript at: http://www.omicsonline.org/submission 